\title{
Assistência de enfermagem na terapêutica paliativa direcionada ao controle de sintomas
}

RESUMO | Objetivo: buscar na literatura científica o que as evidências apontam sobre a assistência da enfermagem ao paciente em cuidados paliativos direcionada ao controle de sintomas. Método: Revisão integrativa da literatura realizada nas bases de dados Lilacs, Scielo, Medline e periódicos da Capes, cujo corpus da análise foi de 8 artigos. Foram incluídos artigos disponíveis nos idiomas inglês, português e espanhol, publicados nos últimos 5 anos, disponíveis na íntegra e gratuitamente. Resultados: Os artigos selecionados apresentaram características diversas, possuindo populações alvo, amostras, finalidades e métodos variados. Após análise dos dados foram encontradas informações que se complementaram acerca do tema, possibilitando apresentação dos resultados em duas categorias: Manifestações clínicas em pacientes em cuidados paliativos e Assistência de enfermagem no manejo de paciente em cuidados paliativos. Conclusão: A enfermagem tem papel de auxiliar na monitoração dos casos de câncer e intervir nos sintomas físicos e psicológicos através de tratamentos farmacológicos e não farmacológicos.

Palavras-chaves: Sinais e Sintomas; Cuidados Paliativos; Neoplasias; Cuidados de Enfermagem.

\begin{abstract}
Objective: to search in the scientific literature what the evidence points about nursing care for patients in palliative care directed to symptom control. Method: Integrative literature review carried out in the Lilacs, Scielo, Medline and Capes journals databases, whose corpus of analysis was 8 articles. Articles available in English, Portuguese and Spanish, published in the last 5 years, were available in full and free of charge. Results: The selected articles had different characteristics, having target populations, samples, purposes and varied methodological means. After analyzing the data, information was found that complemented each other on the topic, made it possible to present the results in two categories: Clinical manifestations in patients in palliative care and Nursing assistance in the management of patients in palliative care. Conclusion: Nursing has the role of assisting in the monitoring of cancer cases and intervening in physical and psychological symptoms through pharmacological and non-pharmacological treatments.
\end{abstract}

Keywords: Signs and Symptoms; Palliative care; Neoplasms; Nursing care.

RESUMEN | Objetivo: buscar en la literatura científica qué apunta la evidencia sobre los cuidados de enfermería al paciente en cuidados paliativos dirigidos al control de síntomas. Método: Revisión integradora de la literatura realizada en las bases de datos de las revistas Lilacs, Scielo, Medline y Capes, cuyo corpus de análisis fue de 8 artículos. Los artículos disponibles en inglés, portugués y español, publicados en los últimos 5 años, estaban disponibles en su totalidad y de forma gratuita. Resultados: Los artículos seleccionados tenían características diferentes, teniendo poblaciones objetivo, muestras, propósitos y medios metodológicos variados. Luego del análisis de los datos, se encontró información que se complementaba sobre el tema, posibilitó presentar los resultados en dos categorías: Manifestaciones clínicas en pacientes en cuidados paliativos y Asistencia de enfermería en el manejo de pacientes en cuidados paliativos. Conclusión: Enfermería tiene el rol de asistir en el seguimiento de los casos de cáncer e intervenir en los síntomas físicos y psicológicos mediante tratamientos farmacológicos y no farmacológicos. Palabras claves: Signos y sintomas; Cuidados Paliativos; Neoplasias; Atención en enfermería.

\section{Hirla Vanessa Soares de Araújo}

Enfermeira Mestre e Doutoranda pelo Programa Associado de Pós-Graduação em Enfermagem UPE/UEPB. Recife (PE), Brasil. ORCID: 0000-0002-9995-7288

\section{Crisnelly Maria da Silva}

Acadêmica de Enfermagem, Centro Universitário dos Guararapes (UNIFG). Jaboatão dos Guararapes (PE), Brasil.

ORCID: 0000-0002-1377-0368

\section{Steven Wesley da Silva Cunha}

Acadêmico de Enfermagem, Centro Universitário dos Guararapes (UNIFG). Jaboatão dos Guararapes (PE), Brasil.

ORCID: 0000-0002-0270-4971

\section{Thaís Roberta de Melo Silva}

Acadêmica de Enfermagem, Centro Universitário dos Guararapes (UNIFG). Jaboatão dos Guararapes (PE), Brasil.

ORCID: 0000-0002-0823-5125

\section{Camila Abrantes Cordeiro Morais}

Enfermeira. Mestre e Doutoranda Programa Associado de Pós-Graduação em Enfermagem UPE/UEPB. Recife (PE), Brasil.

ORCID: 0000-0003-3780-9340

\section{Simone Maria Muniz da Silva Bezerra}

Enfermeira. Doutora. Docente do Programa Associado de Pós-Graduação em Enfermagem UPE/UEPB. Recife (PE), Brasil.

ORCID: 0000-0002-0974-1409

Recebido em: 26/02/2021

Aprovado em: 14/05/2021
INTRODUÇÃO

ntre todas as doenças crônicas,
uma das mais conhecidas e te-
midas na atualidade é o câncer. Essa patologia é caracterizada pelo crescimento desordenado de células em diferentes locais do corpo, criando assim uma neoplasia, a qual é capaz de espalhar-se pelo organismo, fenômeno conhecido como metástase. Esse processo pode trazer diversos danos à homeostase do indivíduo, acarretando constantemente consequências irreversíveis ${ }^{(1)}$. Muitas vezes o diagnóstico é feito tardiamente, quando o tumor já está avançado, e por esse motivo, observam-se muitos casos em que a terapia curativa 
não apresenta mais o efeito esperado no paciente ${ }^{(2-3)}$.

A incidência do câncer vem aumentando em todo o mundo devido a fatores de risco internos e externos relacionados ao paciente, sendo eles: idade, etnia, hereditariedade, gênero, alimentação inadequada, sedentarismo, sobrepeso, uso de tabaco e bebidas alcoólicas, situação socioeconômica, etc ${ }^{(3-4)}$.

No Brasil, o órgão governamental responsável por todas as políticas integrais da nação relacionadas ao controle e prevenção do câncer é o INCA (Instituto Nacional de Câncer José de Alencar Gomes da Silva). Este instituto determinou para o triênio de 2020/2022 a ocorrência de 625 mil novos $\operatorname{casos}^{(5)}$.

No momento em que a terapia curativa não é mais viável são inseridos os cuidados paliativos, nos quais os profissionais de saúde não tratam mais a doença em si, mas o paciente e sua qualidade de vida ${ }^{(2)}$. Assim, analisa-se que a equipe de saúde trabalhará para manter seu paciente vivo e estável, no entanto, ciente que o processo da morte é algo que precisa ser abordado e aceito, para que haja um melhor prognóstico durante o tratamento ${ }^{(6)}$.

De acordo com a Organização Mundial de Saúde (OMS), definem-se como Cuidados Paliativos (CP) aqueles procedimentos que visam à melhoria da qualidade de vida de pacientes em estado terminal. Estes são direcionados não apenas aos pacientes, mas também às famílias que enfrentam situações relacionadas ao fim do ciclo vital. Estima-se que a cada ano 40 milhões de pessoas necessitam desta assistência, entretanto, apenas 14\% o recebem de forma adequada. Tais medidas destinam-se à prevenção e alívio do sofrimento através do reconhecimento precoce e abordagem terapêutica adequada para a dor e demais problemas físicos, emocionais, psicossociais e espirituais ${ }^{(7-8)}$.

A terapêutica paliativa tem como foco principal o controle dos sintomas, sem finalidade de cura, prolongamento ou redução da sobrevida. Para que a promoção do alívio de tais condições seja efetiva, é preciso realizar uma análise individual do histórico do paciente, tendo em mente que tanto o adoecimento quanto a melhora estão associados diretamente com o aspecto espiritual e psicossocial ${ }^{(9)}$.

Em relação ao profissional da área de enfermagem, nota-se a necessidade que este apresente, além das responsabilidades técnicas ou procedimentais, competência de mediação entre a equipe e a família É importante entender as nuances e preceitos que são pertinentes aos cuidados paliativos, fazendo uso de tais características para auxiliar de forma adequada na reflexão e adesão dos pacientes e de suas famílias aos tratamentos e medidas necessárias ${ }^{(10)}$.

A partir do levantamento de estudos sobre a temática, busca-se responder à questão norteadora: Quais são os principais sintomas observados em pacientes diagnosticados com câncer terminal e quais condutas o enfermeiro deve realizar para que esses sintomas sejam controlados?

Diante da literatura revisada, a pesquisa foi conduzida com o objetivo de buscar na literatura científica o que as evidências apontam sobre a assistência de enfermagem na terapêutica paliativa direcionada ao controle dos sintomas em pacientes oncológicos, assim como retratar as estratégias que foram utilizadas pela equipe de enfermagem, explanadas no decorrer da revisão.

\section{MÉTODO}

Trata-se de uma pesquisa de revisão integrativa, desenvolvida com o propósito de reunir e sintetizar achados de estudos realizados, mediante diferentes metodologias com o intuito de colaborar para o aprofundamento do conhecimento relativo ao tema investigado ${ }^{(11)}$. A partir da questão norteadora, espera-se contribuir de forma efetiva para o desenvolvimento da compreensão que envolve a assistência de enfermagem na terapêutica paliativa.

Foi realizado um levantamento de artigos científicos nas bases de dados: Lilacs via Biblioteca Virtual de Saúde (Bireme), Medline via Biblioteca Virtual de Saúde (Bireme), Scientific Electronic Library Online (Scielo) e nos periódicos da Coordenação de Aperfeiçoamento de Pessoal de Nível Superior (Capes). A coleta de dados ocorreu no período de março à junho do ano de 2020, utilizando-se dos descritores (DeCS) cruzados através do operador booleano "AND" dispostos no Quadro 1.

Obedeceu-se a mesma sequência na inserção dos descritores para as buscas, delimitando-se em cinco anos anteriores ao período estabelecido, com o objetivo de abranger o maior quantitativo de publicações a respeito da temática. Foram incluídos artigos nos idiomas inglês, por-

Quadro 1: Estratégias de busca para a consulta nas bases de dados.

\begin{tabular}{|c|c|}
\hline Cruzamentos em Inglês & Cruzamentos em Português \\
\hline Neoplasms (DeCS) AND Nursing care (DeCS) & Neoplasias (DeCS) AND Cuidados de enfermagem (DeCS) \\
\hline Palliative Care (DeCS) AND Signs and Symptoms (DeCS) & Cuidados Paliativos (DeCS) AND Sinais e Sintomas (DeCS) \\
\hline Nursing care (DeCS) AND Palliative Care (DeCS) & Cuidados de enfermagem (DeCS) AND Cuidados Paliativos (DeCS) \\
\hline Neoplasms (DeCS) AND Palliative Care (DeCS) & Neoplasias (DeCS) AND Cuidados Paliativos (DeCS) \\
\hline Neoplasms (DeCS) AND Signs and Symptoms (DeCS) & Neoplasias (DeCS) AND Sinais e Sintomas (DeCS) \\
\hline $\begin{array}{c}\text { Neoplasms (DeCS) AND Nursing care (DeCS) AND Palliative Care (DeCS) } \\
\text { AND Signs and Symptoms (DeCS) }\end{array}$ & $\begin{array}{c}\text { Neoplasias (DeCS) AND Cuidados de Enfermagem (DeCS) AND Cuidados } \\
\text { Paliativos (DeCS) AND Sinais e Sintomas (DeCS) }\end{array}$ \\
\hline
\end{tabular}


tuguês e espanhol, sem restrição de país de publicação, publicados nos últimos cinco anos e disponíveis na íntegra e gratuitamente. Foram excluídos artigos que não respondessem à pergunta norteadora do estudo.

A leitura seletiva dos artigos foi realizada, inicialmente, com análise do título e resumo. Os artigos duplicados foram registrados apenas uma vez. Para garantir o registro conjunto de informações relevantes ao tema foi utilizado instrumento próprio para análise dos artigos, o qual incluía dados de identificação (período publicado, formação e instituição à qual os autores estão vinculados, idioma e país), aspectos metodológicos dos estudos (delineamento da pesquisa, amostragem e tratamento dos dados) e principais resultados e conclusões. Em seguida, realizou-se análise detalhada dos artigos, considerando o rigor e as características dos mesmos, seguindo-se ao registro das informações elencadas no instrumento. As informações foram interpretadas, resumidas e organizadas em quadro síntese, comparando-se os resultados, e finalizadas com proposições acerca dos estudos.

Os artigos selecionados foram submetidos à classificação do nível de evidência constituída por sete níveis: I) evidências obtidas de revisão sistemática ou metaanálise de ensaios clínicos randomizados, controlados ou diretrizes clínicas baseadas em

\section{Figura 1- Fluxograma do processo de seleção dos estudos. Recife, PE, Brasil, 2020.} Adaptado do PRISMA-ScR ${ }^{(13)}$.

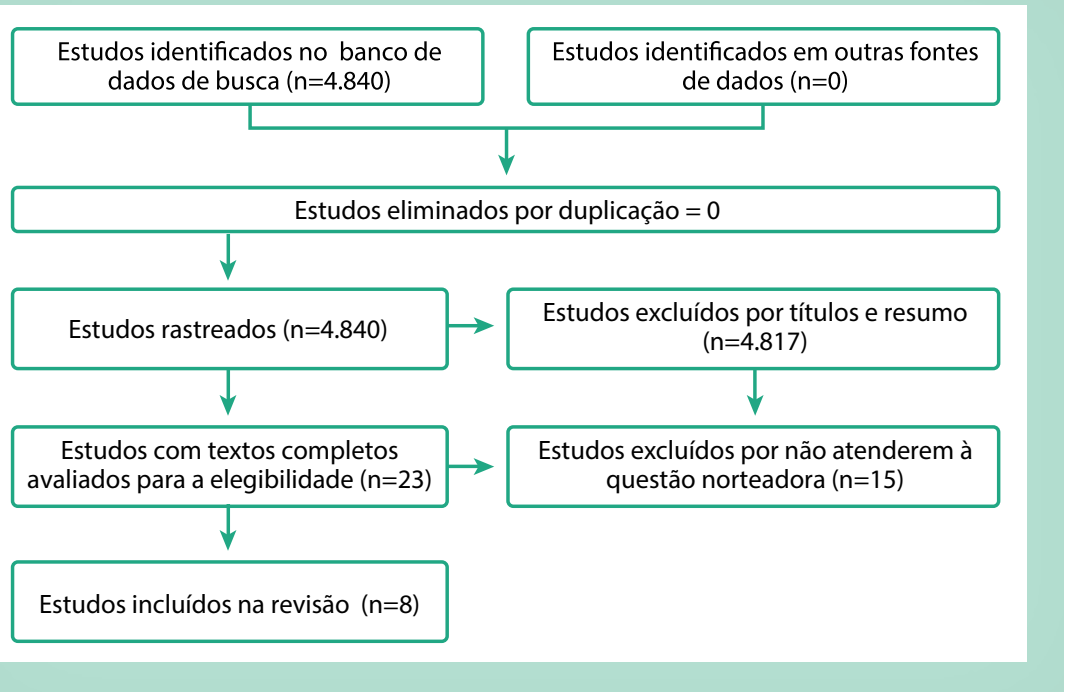

revisões sistemáticas de ensaios clínicos randomizados controlados; II) evidências oriundas de pelo menos um ensaio clínico randomizado, controlado, bem delineado; III) evidências provenientes de ensaios clínicos, sem randomização, bem delineados; IV) evidências obtidas de estudos de coorte e caso controle, bem delineados; $\mathrm{V}$ ) evidências que tiveram origem de revisão sistemática de estudos descritivos e qualitativos; VI) evidências oriundas de um único estudo descritivo ou qualitativo e; VII) evidências provenientes de opinião de autoridades ou relatório de comitês de especialistas. Segundo esta classificação, os níveis 1 e 2 são considerados evidências fortes, 3 e 4 moderadas e 5 a 7 fracas ${ }^{(12)}$.

\section{RESULTADOS}

Inicialmente, foram encontrados 4.840 artigos científicos, utilizando os descritores e termos relacionados de forma direta, respeitando os critérios de inclusão e elegibilidade. Após a leitura dos títulos, foram excluídos 4.744 artigos por não respeitarem os critérios de inclusão, e mais 39 artigos após a leitura do resumo, pelo mesmo motivo. Em seguida, foi realizada a leitura de 23 publicações na íntegra. Destas, 15 foram excluídas por não responderem à questão norteadora da revisão integrativa. Assim, foi obtido um total de 8 artigos científicos selecionados que estavam voltados para os cuidados de enfermagem no controle de sintomas em cuidados paliativos oncológicos.

Figura 2: Síntese dos artigos sobre os cuidados de enfermagem no controle de sintomas de pacientes em cuidados paliativos. Recife, PE, Brasil, 2020.

Título/Ano de publicação

Diagnóstico de enfermagem em cuidados paliativos oncológicos: Revisão integrativa (2019) ${ }^{(14)}$

\section{Método/Nível de evidência}

Revisão integrativa de literatura (V)

\section{Principais Resultados}

Os três diferentes diagnósticos de enfermagem (risco, promoção de saúde e foco no problema) são utilizados para proporcionar uma melhor assistência no controle de sinais e sintomas de pacientes com cuidados paliativos, pois através deles, as medidas de intervenção são aplicadas de forma mais eficaz;

Segundo o estudo, os sintomas mais apresentados pelos pacientes foram: dor $(76,5 \%)$, aflição $(49,8 \%)$, insônia $(34,1 \%)$ e delírio $(25,1 \%)$. E os principais diagnósticos de enfermagem baseados nos sintomas foram: Dor crônica, Sofrimento Espiritual, Dor Aguda e Ansiedade relacionada à morte. 
Evidence-Based palliative care approaches to non-pain physical symptom management in cancer patients; $(2018)^{(15)}$
Revisão integrativa da literatura (V)
Outcomes From a Patient-Centered, Interprofessional, Palliative Consult Team in Oncology; $(2018)^{(16)}$
Traz alguns dos principais sintomas físicos que podem ser tratados com terapia não dolorosa, sendo eles: náuseas e vômitos, constipação, diarreia anorexia e caquexia, fadiga, delírio e dispneia. Sendo náuseas e vômitos os sintomas mais preocupantes pois podem fazer com que o paciente queira abandonar o tratamento.

Seguindo as orientações do Edmonton Symptom Assessment System, foram analisados os principais sintomas: dor, fadiga, depressão, ansiedade, além de demais sintomas relacionados;

Todos os sintomas foram graduados como leve, moderado, grave, em três ocasiões diferente: triagem, consulta inicial, e na alta. Os dados foram comparados estatisticamente, para compreender como o cuidado multiprofissional auxilia em amenizar os sintomas acima citados;

No tempo do estudo, o índice geral dos sintomas foi diminuindo de forma significativa em uma grande porcentagem dos pacientes, depois dos devidos cuidados paliativos.

Sintomas de ansiedade, depressão e desesperança são comuns em pacientes com câncer avançado, podendo afetar a adesão ao tratamento e a qualidade de vida; Tratamentos farmacológicos, complementados com não farmacológicos são mais eficientes quando se tem apoio dos enfermeiros qualificados que acompanham o paciente,

\section{Psychological symptoms in \\ Revisão integrativa de} advanced cancer $(2018)^{(17)}$ literatura (V)

Symptom Management and
$\begin{aligned} & \text { Palliative Care for Patients with } \\ & \text { Cancer }(2017)^{(18)}\end{aligned}$
macological pain treatment in cancer patients $(2017)^{(19)}$

\section{Revisão integrativa de literatura (V)}

Para otimizar os cuidados paliativos, os enfermeiros precisam ter ótimas capacidades de avaliação e gerenciamento dos determinados perfis de sintomas dinâmicos complexos;

Acesso a informações dos diversos planos de cuidado para controle de sintomas (por exemplo, Edmonton Symptom Assessment Scale, Memorial Symptom Assessment Scale, Patient-Reported Outcomes Versiono fthe Common Terminology Criteria for Adverse Events, entre outros) que demonstraram itens valiosos para se ter um olhar mais completo e lidar com os possíveis sintomas do paciente.

Traz a comunicação como sendo uma ação necessária para o alívio de sintomas psicológicos;

Outras condutas encontradas para alívio dos sintomas de pacientes oncológicos, além do uso de analgésicos, foram: mudanças de decúbito, uso de terapia de aplicação de calor, massoterapia, musicoterapia, reiki, incentivo à deambulação, carinho, conforto, sensibilidade e proximidade com a equipe e com a família.

Identificar as melhores práticas baseadas em evidências tem sido uma questão desafiadora, visto que a população de pacientes gravemente doentes é bastante vunerável e heterogênea;

Symptom Control at the End of Life $(2016)^{(20)}$
Revisão integrativa de literatura (V)
É essencial para o gerenciamento dos sintomas no final da vida que as intervenções aconteçam sob medida para abordar a causa do sofrimento de forma alinhada com o modo que o paciente desejar levar o prognóstico.
Escala multidimensional na avaliação da dor e sintomas de idosos em cuidados paliativos $(2016)^{(21)}$
Estudo quantitativo de corte transversal (VI)
Para avaliar a dor e outros sintomas, utilizou-se o instrumento Edmonton Symptom Assessment System (ESAS-r). Os sintomas com maior evidência e em maior intensidade foram ansiedade, dor e cansaço. Aqueles que não representaram valores significativos foram: prisão de ventre, diarreia, incontinência urinária e polaciúria;

Fonte: elaborado pelos autores.

A Figura 1 exemplifica o processo de seleção dos artigos através de um fluxograma, seguindo os critérios de elegibilidade já citados anteriormente. Observou-se que não existiam alguns descritores que apresentassem relação direta com o tema, portanto foi necessário utilizar descritores já conhecidos que fossem sinônimos do tema em questão, para que o objetivo da pesquisa fosse alcançado com sucesso.

Dos 8 artigos escolhidos para o corpus da análise, 5 estavam disponíveis na língua inglesa, e 3 na língua portuguesa, sendo 6 revisões integrativas de literatura e 2 estudos transversais. Em relação aos locais onde as pesquisas foram desenvolvidas, 4 estudos foram realizados nos Estados Unidos da América, 3 no Brasil e 1 no Canadá. A figura 2 elenca os nomes dos artigos, seus autores, ano de publicação, país onde foram elaborados, representação da amostra, e os resultados que se mostraram mais relevantes. 
DISCUSSÃO

Os artigos selecionados apresentaram características diversas, possuindo populações alvo, amostras, finalidades e meios metodológicos variados. Após análise dos dados, foram encontradas informações que se complementaram acerca do tema, possibilitando assim uma revisão focada nos principais sintomas apresentados por pacientes oncológicos, bem como as medidas de controle disponíveis. O estabelecimento destas considerações possibilitou a apresentação dos resultados em duas categorias: Manifestações clínicas em pacientes em cuidados paliativos e Assistência de enfermagem no manejo de paciente em cuidados paliativos.

Categoria 1: Manifestações clínicas em pacientes em cuidados paliativos

Os pacientes oncológicos que estão em CP apresentam determinadas manifestações clínicas que merecem destaque, sendo alguns dos sinais e sintomas mais frequentes: dor, inapetência, dispneia, náuseas e vômitos, constipação intestinal, insônia, diarreia, fadiga, depressão e ansiedade ${ }^{(1,22)}$.

A dor é uma manifestação clínica que pode ser detectada em cerca de $90 \%$ dos pacientes em cuidados paliativos, causando desconforto e sofrimento intenso ${ }^{(21,23)}$. Esse sintoma pode fazer com que o paciente desencadeie outras manifestações como dispneia, delírio e ansiedade ${ }^{(14,19)}$.

A inapetência (ou perda de apetite) é um dos sintomas mais referenciados por pacientes com câncer avançado. Geralmente, este sintoma está ligado a um quadro de anorexia, que se apresenta como uma aversão a comida, e caquexia, que é uma síndrome multifatorial que causa a perda contínua da massa muscular esquelética podendo levar à atrofia ${ }^{(20)}$. Esses três sintomas (inapetência, anorexia e caquexia) muitas vezes aparecem juntos em um mesmo paciente, levando a um grave quadro de desnutrição e fraqueza ${ }^{(15)}$.
A dispneia (ou desconforto respiratório) é um sintoma observado em até $79 \%$ dos pacientes no final da vida e é proveniente de vários fatores, podendo ser: fisiológicos, psicológicos, sociais e ambientais $^{(15,24)}$

Ainda, náuseas e vômitos apresentam-se como uma das principais preocupações para os pacientes em tratamento de câncer, pois possuem capacidade para interferir diretamente na qualidade de vida dos mesmos além de prejudicar a adesão ao tratamento. A náusea pode se relacionar a outros sintomas como sudorese fria, taquicardia e diarreia. Pacientes com câncer que estão fazendo uso de quimioterapia são quatro vezes mais propensos a desenvolver náuseas e vômitos durante o primeiro ciclo do tratamento ${ }^{(15,18)}$.

Em relação à constipação, a forma mais comum observada foi a relacionada com o uso de opioides, pois estas medicações unem-se aos receptores neuronais entéricos perturbando as coordenações destinadas ao peristaltismo, diminuindo assim a motilidade intestinal ${ }^{(15,20)}$. A queixa de diarreia está ligada a muitos tipos de câncer, porém, é um sintoma que também pode ser derivado do tratamento de quimioterapia, radioterapia e imunoterapia ${ }^{(15)}$.

A fadiga também é uma manifestação muito comum em pacientes em tratamento com quimioterapia e radioterapia, geralmente devido aos efeitos colaterais dos fármacos, porém, também pode ser proveniente de alterações metabólicas, como por exemplo, o hipotireoidismo ${ }^{(15)}$.

Já os transtornos depressivos têm se tornado cada vez mais frequentes em pacientes em $\mathrm{CP}$, podendo atingir até $60 \%$ dos casos, esse sintoma torna-se um agravante para que o bem-estar geral do paciente caia drasticamente ${ }^{(18,20)}$. Podem estar diretamente relacionados a fatores físicos, biológicos, psicológicos e/ou sociais, portanto é imprescindível que o enfermeiro procure saber qual foi o motivo para o desencadeamento da situação depressiva $^{(16)}$.
No tocante à ansiedade, esta pode ser causada por diversos fatores socioeconômicos ou pessoais. Doenças crônicas, como o câncer, tendem a aumentar a incidência de sintomas psicológicos. Geralmente esse sintoma ocorre entre $20 \%$ a $60 \%$ dos pacientes em cuidados paliativos oncológicos ${ }^{(17,19)}$.

A insônia é um problema frequente em diversos tipos de câncer, independentemente da idade, todavia, aproximadamente $75 \%$ de pacientes idosos com câncer tem problemas relacionados à insônia. Essa interrupção do sono pode causar outros sintomas ao paciente, como fadiga, depressão e/ou outros problemas psíquicos ${ }^{(17,21)}$.

Categoria 2: Assistência de enfermagem no manejo de paciente em cuidados paliativos.

O enfermeiro, juntamente com toda a equipe multidisciplinar responsável pelo paciente, deve estar preparado para fornecer a melhor assistência possível, sempre atendendo as suas necessidades ${ }^{(25)}$. Os cuidados de enfermagem ao paciente em CP se relacionam tanto com o manejo medicamentoso (uso de fármacos para aliviar os sintomas) como com condutas não farmacológicas ${ }^{(19)}$.

Em busca de uma melhora ou alívio da dor, por exemplo, utilizam-se terapias medicamentosas, como: analgésicos (geralmente opioides) corticosteroides, antidepressivos, anticonvulsivantes, analgesia não-opioide, bisfosfonato, entre outros ${ }^{(18)}$. Já a terapia não farmacológica pode ser aplicada através de mudança de decúbito, terapia de calor, incentivo à deambulação, comunicação interpessoal, uso de coxins, musicoterapia, reiki, massoterapia, carinho, apoio emocional e proximidade familiar e multidisciplinar ${ }^{(19)}$.

Já para outros sintomas, como inapetência, anorexia e caquexia, pedem que diversas abordagens sejam efetivadas para que a recuperação aconteça, dentre elas: tratamentos não farmacológicos que incluem uma melhoria na dieta e a prática de atividades físicas, ambas 
as tarefas orientadas por profissionais qualificados; o método terapêutico farmacológico é variado, mas segundo as informações coletadas, as medicações dronabinol e acetato de magestrol (ambas aprovadas pelo FDA-Food and Drug Administration - para o tratamento de anorexia), seguindo rígida orientação médica graças a seus efeitos colaterais, são amplamente utilizados ${ }^{(15,26)}$.

O tratamento para a dispneia consiste em utilizar a oxigenoterapia e fármacos opioides $^{(15)}$, sendo os opioides mais eficazes nos quadros de pacientes dispneicos nos últimos dias de vida ${ }^{(20)}$. Para a utilização da terapia adequada, deve-se primeiramente buscar a causa base da dispneia, pois pode ser uma causa primária ou associada com outra alteração, como por exemplo, a dor ${ }^{(14)}$. Existem poucas provas do alívio desse sintoma através de medidas não farmacológicas ${ }^{(15)}$.

Já o manejo das náuseas e dos vômitos, a terapêutica envolve a administração de antieméticos para prevenção e tratamento da êmese. Todavia, é necessário descobrir primeiramente a etiologia do sintoma para dar seguimento ao tratamento adequado, por exemplo, quando a constipação é a etiologia de ambos, é indicado o uso de laxantes para aliviar os sintomas. Existem também outros fatores de risco individuais que elevam a chances de desenvolver este sintoma, são eles: sexo feminino, idade inferior a 50 anos, alta ingestão de álcool, tratamento quimioterápico, entre outros ${ }^{(15,18)}$.

É comum observar casos de constipação intestinal em pacientes oncológicos, muitas vezes sendo ocasionadas pelo uso de opioides. Neste caso, a profilaxia deve envolver o início de um regime de opioides e deve-se evitar o uso de laxantes, pois podem piorar o quadro. Para melhor abordagem, todo histórico do paciente precisa ser levado em consideração, em busca de mudanças como: atividades físicas diminuídas, dieta alterada, distúrbios de motilidade, distúrbios endócrinos e metabólicos, entre outros. A escolha do tratamento deve também envolver as características das fezes, se estas forem mais líquidas, o uso de um supositório se faz eficiente, se forem mais sólidas, um enema mostra-se como mais apropriado(15,20).

$\mathrm{Na}$ diarreia, é necessário que informações como: o início, a frequência e as características das fezes, sejam analisadas para melhor manejo da situação. Estratégias não farmacológicas como reidratação oral e mudanças na alimentação, bem como a ingestão de microrganismos vivos como probióticos e lactobacillus são bem aceitas. Após a quimioterapia, é comum a indicação de loperamina oral 4 mg como a primeira dose e $2 \mathrm{mg}$ a cada 4 horas (dose máxima de $16 \mathrm{mg} /$ dia) para a diminuição da motilidade intestinal e redução dos sintomas adjacentes ${ }^{(15)}$.

A melhora da fadiga pode ser obtida através de fármacos psicoestimulantes, esteroides (especialmente para pessoas que apresentam quadro anoréxico) e metilprednisona $^{(20)}$. Também pode ser aplicada a terapia não farmacológica, feita com o auxílio de terapias cognitivas, apoio social e psicológico e de métodos para evitar o gasto energético desses pacientes ${ }^{(15)}$.

Para os sintomas da depressão ou transtorno depressivo, é necessário o equilíbrio entre o tratamento farmacológico (especialmente com antidepressivos, ou derivados) e a psicoterapia, para que o paciente realmente consiga se estabilizar. A depressão pode acarretar outros sintomas, por isso é imprescindível que o enfermeiro esteja sempre perto do paciente, dando total auxílio nas demais terapias, e ter um olhar crítico e holístico para identificar as melhores condutas a fim de proporcionar ao paciente uma melhor qualidade de vida ${ }^{(17,18,20)}$.

No quadro de ansiedade observa-se que por mais que a intervenção farmacêutica (em especial, o uso de ansiolíticos) possa ser uma das melhores medidas para controle do sintoma, o enfermeiro precisa ter um olhar diferenciado, prezando métodos como relaxamento, escuta ativa, modos educados, uma boa comunicação e possível encaminhamento para um psicólogo. É importante ressaltar que o cuidado e tratamento da ansiedade deve ser focado no paciente e nas suas necessidades básicas, medos e aflições, para que o paciente compreenda sua situação, a aceite, havendo assim uma meIhor adesão ao tratamento ${ }^{(14,16,17)}$.

No caso da insônia, se faz necessário um tratamento específico para que o paciente se sinta o mais confortável possível, auxiliando na diminuição do sintoma. $\mathrm{O}$ enfermeiro que assiste o paciente insone deve analisar todo esse contexto, além de supervisionar a prescrição e administração de fármacos para auxiliar o paciente a regularizar seu sono, caso prescrito pelo médico. Entretanto, os medicamentos não são a única opção para melhorar o padrão de sono, terapias para relaxamento e apoio psicológico também são importantes ferramentas utilizadas ${ }^{(18,21)}$.

Analisando o contexto das diversas fisiopatologias e tratamentos, observa-se a importância de um trabalho engajado da equipe multidisciplinar, pois todos os profissionais podem juntos elaborar planos adequados para ajudar o aceitamento da atual situação, seja para o paciente ou para sua família, especialmente as mudanças que serão acarretadas pela doença em si. Assim, nota-se que o enfermeiro dentro da equipe multiprofissional tem um papel vital no cuidado a esse paciente, sendo o profissional de contato mais próximo, criando um forte vínculo de confiança, possibilitando a identificação de maneira eficiente das melhorias que são necessárias naquele momento, visando a melhor qualidade de vida ${ }^{(27-28)}$.

Por ser um setor onde necessita-se de um alto nível de assistência no decorrer de todo processo da terapêutica com o paciente, a Oncologia requer cuidados específicos de enfermagem, requisitando habilidades peculiares para lidar com essa realidade. Essas competências não são apenas referentes ao âmbito técnico, mas principalmente relacionadas à parte de relacionamento e afeição para com o paciente, considerando o indivíduo como um todo ${ }^{(29-30)}$. Essa análise de enfermagem se aplica também para o 
controle dos sintomas dos pacientes, pois não basta lidar com a sintomatologia de forma isolada, é necessário ter uma visão holística do mesmo, para que haja um melhor bem-estar e dignidade do usuário do serviço ${ }^{(19,29,31)}$.

Assim, por conta da alta complexidade dos cuidados que se devem ter com esses pacientes, observa-se que o enfermeiro precisa ter um bom domínio das suas habilidades teóricas e práticas, para que os cuidados paliativos sejam realmente eficientes. A avaliação desses cuidados realizados pelos profissionais de enfermagem é essencial para que se haja uma real continuação do cuidado com os pacientes em $\mathrm{CP}^{(32-33-34)}$.

Alguns dos estudos analisados mostraram que a maior parte dos cuidados oncológicos, em especial CP são relacionados ao ambiente hospitalar em diversos lugares do mundo, em especial no Brasil. Existe uma escassez enorme com relação ao diagnóstico precoce da doença e ao seu devido rastreamento. Assim, observa-se que a enfermagem em todos os seus níveis de atendimento, desde a atenção primária até a mais especializada, tem um papel importante para auxiliar a monitorar todos os casos. Assim fazendo, oferecem-se mais oportunidades aos pacientes oncológicos e seus devidos tratamentos, além de incentivo à sua autogestão, e se possível, a diminuição da probabilidade de adentrar em cuidados paliativos $^{(18,35,36)}$.

Entretanto, diversos estudos analisados mostram que ainda existe um déficit muito grande na formação de profissionais de enfermagem na área de oncologia, fator que influencia diretamente no cuidado dado ao paciente. Enquanto alguns estudos mostram que a enfermagem é a área profissional que mais produz artigos e demais produções científicas relacionadas aos cuidados paliativos nas principais plataformas de pesquisa ${ }^{(37-38)}$, na prática outros estudos revelam que os enfermeiros não recebem instrução adequada que os preparem para com os pacientes em CP. Profissionais recém chega-
Enquanto alguns estudos mostram que a enfermagem é a área profissional que mais produz artigos e demais produções científicas relacionadas aos cuidados paliativos nas principais plataformas de pesquisa, na prática outros estudos revelam que os enfermeiros não recebem instrução adequada que os preparem para com os pacientes em CP. dos nessa área sentem que a preparação nos diversos níveis acadêmicos não fora suficiente, na maioria das vezes a prática e a pesquisa trazem uma melhor base para eles. Portanto, é necessário que os diversos cursos de enfermagem tenham disciplinas e preparações mais adequadas para que os enfermeiros recém-formados tenham mais segurança ao entrar nesses setores ${ }^{(39)}$.

Por ser a classe de profissionais que está em contato direto com os pacientes, a "arte de cuidar" dos enfermeiros mostra-se como a linha de frente para que os cuidados paliativos alcancem os resultados esperados, esperando-se que os sintomas estejam sempre estáveis para que o usuário se sinta bem de forma constante, ou na maior parte do tempo. Portanto, analisa-se que enfermeiros devidamente capacitados, pautados no respeito, humanização e acolhimento, são uma ferramenta de vital importância para o melhor tratamento dos pacientes que estão em uso dos cuidados paliativos e para o controle dos seus sintomas ${ }^{(38-40)}$.

\section{CONCLUSÃO}

A pesquisa permitiu identificar os sintomas mais frequentes em pacientes em cuidados paliativos e as condutas do enfermeiro para que haja um bom controle deles. Percebe-se que os usuários do serviço de saúde necessitam de um cuidado amplo, complexo e dirigido para sua própria situação, pois cada um deles refere diferentes graus do avanço do câncer e de como isso influencia a atual sintomatologia do enfermo.

Nesse cenário, o enfermeiro é um dos protagonistas do cuidado ao paciente em cuidados paliativos e seus sintomas, sendo o profissional que está em contato direto com o usuário. O conhecimento teórico e prático desses profissionais faz com que se haja um cuidado integral e holístico com o paciente, levando em conta toda a sintomatologia não apenas física, mas também emocional, psíquica, social, espiritual. 


\section{Referências}

1. BRASIL. Ministério da Saúde. $A B C$ do câncer: abordagens básicas para 0 controle do câncer / Instituto Nacional de Câncer José Alencar Gomes da Silva; organização Mario Jorge Sobreira da Silva. - 5. ed. rev. atual. Amp. I. - Rio de Janeiro: Inca, 2019. Disponível em: https://www.inca.gov.br/sites/ufu.sti.inca. local/files//media/document/livro-abc-5-edicao_1.pdf

2. Andrade GB, Pedroso VSM, Weykamp JM, Soares LS, Siqueira HCH, Yasin JCM. Cuidados Paliativos e a Importância da Comunicação entre o Enfermeiro e Paciente, Familiar e Cuidador. Rev Fund Care Online. 2019; 11(3): 713-7. 10.9789/2175-5361.2019.v11i3.713-717.

3. Visentin A, Mantovani MF, Kalinke LP, Boller S, Sarquis LLM. A terapêutica paliativa em adultos com câncer: um estudo transversal. Rev Bras Enferm. 2018; 71(2): 252-8. http://dx.doi.org/10.1590/0034-7167-2016-0563.

4. INSTITUTO NACIONAL DO CÂNCER (INCA). Estimativa 2020: incidência de câncer no Brasil - Rio de Janeiro: INCA, 2019.

5. INSTITUTO NACIONAL DO CÂNCER (INCA). Números do câncer. Estatística do câncer. BRASIL, ano 2020, 5 fev. 2020.

6. Meneguin S, Matos TDS, Ferreira MLSM. Percepção de pacientes oncológicos em cuidados paliativos sobre qualidade de vida. Rev Bras Enferm. 2018; 71(4): 1998-2004. DOI: http://dx.doi.org/10.1590/0034-7167-2017-0360.

7. World Health Organization. Cancer. World Health Organization, 2020.

8. World Health Organization. Palliative care. World Health Organization, 2020. Disponível em: https://www.who.int/cancer/palliative/en/\#. Acesso em: 15 mar. 2020.

9. Markus LA, Betiolli SE, Souza SJP, Marques FR, Migoto MT. A atuação do enfermeiro na assistência ao paciente em cuidados paliativo. Revista Gestão e Saúde. 2017; 17 (Supl 1): 71-81.

10. Cavalcanti IMC, Oliveira LO, Macêdo LC, Leal MHC, Morimura MCR, Gomes ET. Princípios dos cuidados paliativos em terapia intensiva na perspectiva dos enfermeiros. Rev Cuid. 2019; 10(1): e555. https://doi.org/10.15649/cuidarte. v10i1.555

11. Mendes KDS, Silveira RCCP, Galvão CM. Integrative literature review: a research method to incorporate evidence in health care and nursing. Texto Contexto Enferm, 2008; 17(4): 758-64. DOI: 10.1590/50104-07072008000400018 12. Stillwell SB, Fineout-Overholt E, Melnyk BM, Williamson KM. Searching for the Evidence. Am J Nurs, 2010; 110(5): 41-7.

13. Tricco AC, Lillie E, Zarin W, O'Brien KK, Colquhoun H, Levac D, et al. PRISMA extension for scoping reviews (PRISMA-ScR): Checklist and explanation. Ann Intern Med. 2018;169(7):467-73. doi: https://doi.org/10.7326/M18-0850.

14. Passarelles DMA, Rios AA, Santana RF. Diagnósticos de enfermería en cuidados paliativos oncológicos: revision integrativa. Enferm glob. 2019; 18(55): 579-611. http://dx.doi.org/10.6018/eglobal.18.3.345201

15. Hagmann C, Cramer A, Kestenbaum A, DurazoC, Downey A, Russel M et al. Evidence-based Palliative Care Approaches to Non-pain Physical Symptom Management in Cancer Patients. Semin Oncol Nurs. 2018; 34(3): 227-240. https://doi.org/10.1016/j.soncn.2018.06.004

16. Feldstain A, Bultz BD, Groot JM, Abdul-Razzak A. Outcomes From a Patient-Centered, Interprofessional, Palliative Consult Team in Oncology. J Natl Compr Canc. Netw. 2018; 16(6): 719-26. 10.6004/jnccn.2018.7014

17. Bail JR, Traeger L, Pirl WF, Bakitas MA. Psychological Symptoms in Advanced Cancer. Semin Oncol Nurs. 2018; 34(3): 241-51. 10.1016/j.soncn.2018.06.005. 18. Yates P. Symptom Management and Palliative Care for Patients with Cancer. Nurs Clin North Am. 2017; 52(1): 179-191. 10.1016/j.cnur.2016.10.006 19. Oliveira Junior NJ, Oliveira SBS, Migowski ER, Riegel F. Nurses' role in the non-pharmacological pain treatment in cancer patients. Rev Dor. 2017; 18(3): 261-5. 10.5935/1806-0013.20170112

20. Kreher M. Symptom Control at the End of Life. Med Clin North Am. 2016; 100(5): 1111-22. 10.1016 / j.mcna.2016.04.020

21. Faller JW, Zilly A, Moura CB, Brusnicki PH. Escala Multidimensional na Avaliação da Dor e Sintomas de Idosos em Cuidados Paliativos. Cogitare Enferm. 2016; 21(2): 01-10.

22. BRASIL. Ministério da Saúde. Instituto Nacional de Câncer. Cuidados paliativos oncológicos: controle de sintomas. Rio de Janeiro: INCA, 2001. Disponível em: https://bvsms.saude.gov.br/bvs/publicacoes/inca/manual_cuida- dos_oncologicos.pdf.

23. Gayoso MV, Avila MAG, Silva TA, Alencar RA. Avaliação do nível de conforto de cuidadores de pacientes com câncer em cuidados paliativos. Rev Latino-Am Enfermagem. 2018; 26: e3029. DOI: 10.1590/1518-8345.2521.3029. 24. Alves ARMV, Pina PSRS. Dispneia em cuidados paliativos: registros de enfermagem e a autoavaliação da dispneia. Rev Enf Ref Coimbra. 2018; 16: 53-62. http://dx.doi.org/10.12707/RIV17075.

25. Silva RS, Santos RD, Evangelista CLS, Marinho CLA, Lira GG, Andrade MS. Atuação da Equipe de Enfermagem sob a ótica de familiares de pacientes em cuidados paliativos. Rev Min Enferm. 2016; 20: e983. 10.5935/1415-2762.20160053

26. Hui D, Dev R, Bruera E. The last days of life: symptom burden and impact on nutrition and hydration in cancer patients. Curr Opin Support Palliat Care. 2015; 9(4): 346-54. https://dx.doi.org/10.1097/SPC.0000000000000171

27. Gomes MIG. Cuidados Paliativos: relação entre a equipe de enfermagem, pacientes oncológicos e seus familiares. Rev Rede cuid saúde. 2019; 13(2): 60-70.

28. Vieira TA, Oliveira M, Martins ERC, Costa CMA, Alves RN, Marta CB. Palliative care to cancer client: the nursing student's perception. Rev Fund Care online. 2017; 9(1): 175-80. 10.9789/2175-5361.2017.v9i1.175-180.

29. Franco ME, Salvetti MG, Donato SCT, Carvalho RT, Franck EM. Percepção de dignidade de pacientes em cuidados paliativos. Texto contexto - enferm. 2019; 28: e20180142. http://dx.doi.org/10.1590/1980-265x-tce-2018-0142

30. Lins FG, Souza SR. Formação dos enfermeiros para o cuidado em oncologia. Rev Enferm UFPE online. 2018; 12(1): 66-74. http:/ /doi.org/10.5205 / 1981-8963-v12i01a22652p66-74-2018.

31. Stube M, Cruz CT, Benetti ERR, Gomes JS, Stumm EMF. Percepções de enfermeiros e manejo da dor de pacientes oncológicos. Rev Min Enferm. 2015; 19(3): 696-703. http://www.dx.doi.org/10.5935/1415-2762.20150053.

32. Agra G, Formiga NS, Oliveira SHS, Sousa ATO, Soares MJGO, Costa MML. Indicadores psicométricos do saber e o fazer de enfermeiros nos cuidados paliativos à pessoa com ferida tumoral. Aquichan. 2018; 18(4): 461-76. https:// doi.org/10.5294/aqui.2018.18.4.8

33. Queiroz TA, Ribeiro ACM, Guedes MVC, Coutinho DTR, Galiza FT, Freitas MC. Cuidado Paliativo ao idoso na terapia intensiva: olhar da equipe de enfermagem. Texto contexto - enferm. 2018; 27(1): e1420016. https://doi. org/10.1590/0104-07072018001420016.

34. Sousa ADRS, Silva LF, Paiva ED. Intervenções de enfermagem nos cuidados paliativos em Oncologia Pediátrica: revisão integrativa. Rev Bras Enferm. 2019; 72(2): 531-40. http://dx.doi.org/10.1590/0034-7167-2018-0121.

35. Magalhães B, Fernandes C, Santos C, Lima L, Martínez-Galiano JM. Autogestão das complicações associadas ao tratamento de quimioterapia: uma scoping review. Journal Health NPEPS. 2019; 4(2): 370-404. http://dx.doi. org/10.30681/252610103722

36. Freire MEM, Costa SFG, Lima RAG, Sawada NO. Qualidade de vida relacionada à Saúde de pacientes com câncer em cuidados paliativos. Texto contexto - enferm. 2018; 27(2): e5420016. https://doi.org/10.1590/0104070720180005420016.

37. Rolim DS, Arboit EL, Kaefer CT, Marisco NS, Ely GZ, Arboit J. Produção científica de enfermeiros brasileiros sobre enfermagem e oncologia: revisão narrativa da literatura. Arq Cienc Saúde UNIPAR. 2019; 23(1): 41-7. https://doi. org/10.25110/arqsaude.v23i1.2019.6261.

38. Sanches KS, Rabin EG, Teixeira PTO. Cenário da publicação científica dos últimos 5 anos sobre cuidados paliativos em oncologia: revisão de escopo. Rev esc enferm USP. 2018; 52: e03336. http://dx.doi.org/10.1590/S1980220X2017009103336.

39. Ramirez OJG, Gonzales GMC, Arias EM. Teorías de enfermaria para La investigación y La práctica em cuidado paliativo. Rev Latino am bioet. 2017; 17(1): 60-79. http://dx.doi.org/10.18359/rlbi.1764.

40. Floriano JJ, Schwinden LM, Rosa FFP, Zuffo A, Mayer BLD. 0 processo de adoecer do paciente com câncer em cuidado paliativo. Revista Nursing. 2020; 23(267): 4508-13. https://doi.org/10.36489/nursing.2020v23i267p4502-4513. 